\title{
THE EQUILIBRIUM AND STABILITY \\ PROPERTIES OF MENISCI: THE MEASUREMENT OF SURFACE TENSION BY EXACT METHODS
}

\author{
J. F. PADDAY \\ Research Division, Kodak Limited, Wealdstone, Harrow HA1 4TY, UK
}

\begin{abstract}
The literature concerning the properties of menisci is briefly reviewed and it is shown that numerical analysis using computers has led to the solution of a whole range of meniscus problems. Menisci are classified and their properties defined according to the nature and number of the supporting solid surfaces and of certain shape characteristics. The sizes and shapes of different types of menisci are then obtained by integrating the Laplace-Young equation numerically.

The free energy of the whole meniscus system is derived in terms of the surface area and the potential energy in the gravitational field and the equilibrium and stability given in terms of the first and second differential of the free energy with respect to perturbation. Axisymmetric perturbations only are considered as they are those of lowest energy and hence most damaging and the energy profiles of such perturbed menisci have been obtained by numerical analysis. It is shown that when critical stability is reached the size and shape of a given meniscus become unique theoretically determined properties. These critical properties have been extracted from the tables of shape and size.

Finally it is shown that a further set of unique properties, but at stable equilibrium, may also be extracted from the table. These unique properties form an excellent set of conditions by which surface tension is measured with great precision.
\end{abstract}

\section{INTRODUCTION}

A meniscus is defined as being the curved surface of an interface of a liquid with another fluid, which is supported by at least one solid surface. This definition covers drops, bubbles, liquid bridges, capillary rise and many other systems containing a curve interface.

The study of menisci covers a period of about $180 \mathrm{yr}$ as is seen in Fig. 1. The earlier investigations aimed at solving practical problems such as the size of a drop delivered from a pharmacist's pipette ${ }^{1}$ and theoretical problems associated with the measurement of surface tension. Over the whole period investigations were concerned with four major problems which were describing

(i) the shape of the meniscus,

(ii) its equilibrium properties,

(iii) its critical properties reached at the point of rupture, and

(iv) the dynamic properties describing its movement and break up.

This study is concerned with the first three aims and brings together the previous work leading up to a full theoretical explanation of the equilibrium and critical properties of menisci.

Historically, properties of menisci became quantitative with the simultaneous publication by Young $^{2}$ and de Laplace $^{3}$ of the expression for the pressure change, $\Delta P$, across an interface in terms of the principal radii of curvature $R_{h}$ and $R_{v}$.

$$
\Delta P=\gamma\left(\frac{1}{R_{h}}+\frac{1}{R_{v}}\right)
$$

where $\gamma$ is the interfacial tension.

This equation may be expressed in analytical form as

$$
\pm \frac{\mathrm{d}^{2} z / \mathrm{d} x^{2}}{\left(1+(\mathrm{d} z / \mathrm{d} x)^{2}\right)^{3 / 2}} \pm \frac{\mathrm{d} z / \mathrm{d} x}{x\left(1+(\mathrm{d} z / \mathrm{d} x)^{2}\right)^{1 / 2}}=\rho \frac{g}{\gamma} Z,
$$

where $x$ and $z$ are the horizontal and vertical coordinates, $\rho$ is the relative density of the material within the meniscus surface to the material outside, $g$ the gravitational acceleration.

Equation 2 cannot be integrated in closed form to give the coordinates of the shape of the meniscus. Bashforth and Adams ${ }^{4}$ were the first to obtain shapes of drops and bubbles by integrating eqn 2 approximately, using Taylor Series. More recently, integration has been carried out with high-speed computers by several different methods ${ }^{5-7}$ and the data so produced have led to renewed interest, the solution to many practical problems and the recent large number of publications (Fig. 1).

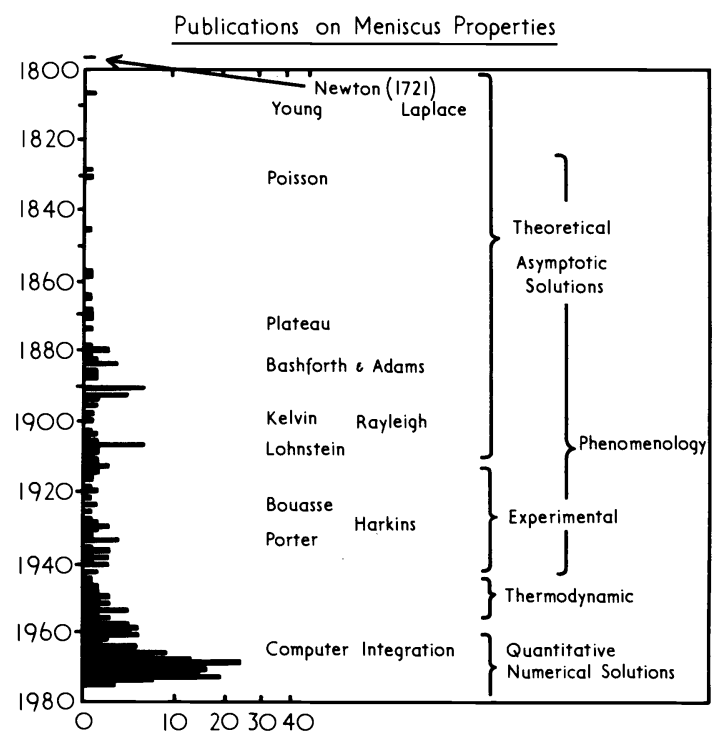

Fig. 1. Number of publications on meniscus studies appearing each year since 1800 . 
TYPES OF MENISCI

The Young-Laplace equation describes a local equilibrium in one part of a meniscus. If integration is to proceed over the whole meniscus then either symmetry or other geometrical constraints must be known and in addition boundary conditions must be specified. It must further be known whether or not the fluid forming the meniscus is distorted by a gravitational, electrical or centrifugal force field.

In this study it is assumed that a uniform gravitational field only acts on the meniscus and that when the density difference of the two fluids is zero, a special limiting case is reached to zero effective gravity. It has been found convenient to distinguish three geometrical types of menisci. They are those with

(a) cylindrical symmetry,

(b) axial symmetry, and

(c) without symmetry.

Cylindrically symmetric menisci possess only one principal radius of curvature $\left(\boldsymbol{R}_{v}\right)$ and represent the special case when $R_{h}$ is infinite as with the Wilhelmy plate. The shape of such menisci may be obtained either by direct integration or by the use of tables of elliptic integrals. ${ }^{8}$

Axisymmetric menisci are common in many areas of study such as bubbles, drops and some types of liquid bridges. Computer solutions of the shapes of such menisci are well known and have been published in tabular form. ${ }^{9,10}$

A number of studies of menisci with asymmetric geometry such as the meniscus of a wedge between two flat plates set at an angle ${ }^{11}$ and between three vertical rods $^{12}$ have been carried out. Such menisci may well lead to solutions of problems associated with the meniscus properties within a porous solid.

\section{Boundary conditions}

Menisci may be further classified according to the number of solid surfaces supporting the curved surface. Here we designate: ${ }^{9,13}$

Bounded menisci are those with only one solid surface supporting the liquid forming the meniscus. In this group fall the meniscus at a Wilhelmy plate, pendant and sessile drops, emergent and sessile bubbles and the meniscus formed by a rod in a free liquid surface.

The reason for describing such menisci as bounded lies in the fact that they are bounded at one extremity by their own envelope or by the free flat surface.

The shape of bounded menisci may be described by a single parameter. Bashforth and Adams used the term

$$
\beta=\frac{\rho g b^{2}}{\gamma}
$$

where $b$ is the radius of a drop or a bubble at its apex. However as this method is unsuitable for use with free surface menisci the author has chosen the rather more general definition. ${ }^{6}$

$$
\beta^{\prime}=\frac{\rho g R_{v}^{2}\left(90^{\circ}\right)}{\gamma}
$$

where $R_{v}\left(90^{\circ}\right)$ is the principal vertical radius of curvature of the meniscus at its neck or narrowest point. $b$ and $\boldsymbol{R}_{v}$ bear, of course, a fixed relationship to each other for a given shape.

\section{A. Captive bubble B. Hanging drop}
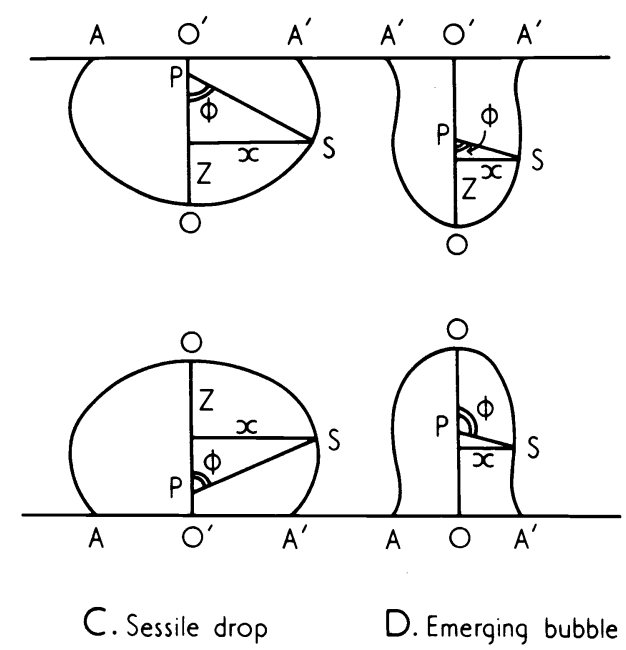

Fig. 2. Types of bonded menisci: (a) Sessile drop, (b) Pendant drop, (c) Sessile bubble, (d) Emergent bubble.

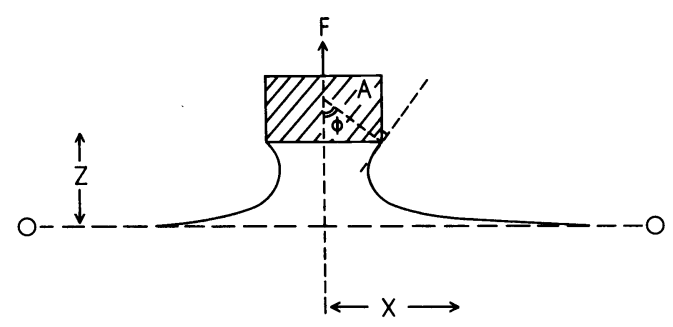

Fig. 3. Rod in free surface meniscus (rifs).

The shapes of drops and bubbles, as is well known, depend on the relative density and the direction of the gravitational force in relation to the supporting surface. In Fig. 2, the more common drop and bubble shapes and in Fig. 3 the rod-in-free-surface (rifs) menisci are shown.

\section{Unbounded menisci}

Unbounded menisci require two supporting solid surfaces to maintain them in position. The most common forms of unbounded menisci are the axisymmetric liquid bridges, examples of which are shown in Fig. 4. The main feature of such menisci is that they require two parameters to describe their shape. In this study we describe the shape of a liquid bridge by the shape factor $\beta^{\prime}$ and the ratio, $R_{v}\left(90^{\circ}\right) / R_{h}\left(90^{\circ}\right)$, of the principal radii of curvature again at the neck of the liquid bridge.

\section{Compound menisci}

These menisci are distinguished from the foregoing by their possession of two or more liquids with a third fluid phase, examples are shown in Fig. 5. These menisci possess interfaces of different chemical composition and hence different interfacial tensions. The simplest is a lens of oil floating on water but an example of a more complex one is that of a rod touching a lens of floating liquid so as to create a compound liquid bridge as shown in Fig. 5. Shape properties are best obtained from existing drop and rifs tables but using special manipulations to bring together the shapes of each part that are at equilibrium with each other. ${ }^{14}$ 
A

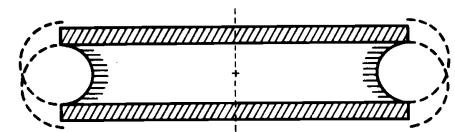

B
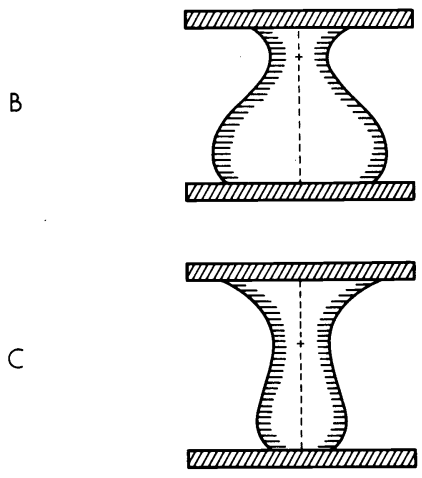

Fig. 4. Unbounded menisci: Liquid bridges.

Compound Menisci

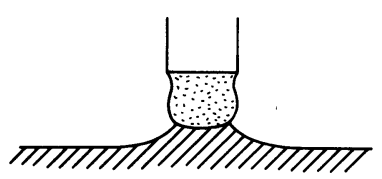

Pendant drop at free surface

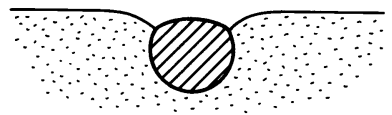

Floating lens

Fig. 5. Compound meniscus: (a) Rod and pendant drop at the free interface of a second immiscible liquid, (b) Floating lens.

\section{MULTIPLE INTERFACE SYSTEMS}

A multiple interface system is one in which two distinct and separate menisci of the same chemical composition are formed and are connected through the supporting solid surface so that their pressures tend to equalise. An example is shown in Fig. 6 and a better known example is that of Plateau ${ }^{15}$-the original soap bubble experiment. Plateau connected two soap bubbles of different sizes to demonstrate the instability of the connected multiple interface system. The equilibrium and stability of each system, though not widely studied, is of great importance in porous media.

\section{THE MENISCUS SUPPORT}

A meniscus requires at least one solid surface to maintain the curved surface at equilibrium in a gravitational field. This solid surface either supports the interface directly or it supports a thin liquid film which holds the meniscus in position. Various types of boundary supports are shown in Fig. 7 and it is evident that a meniscus may
Multiple Interface Systems

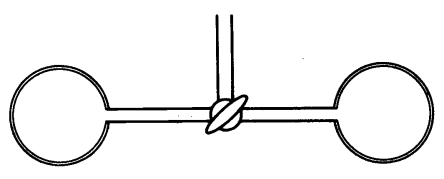

Soap bubbles

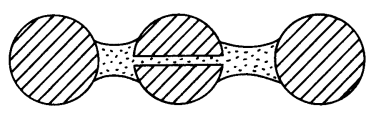

Connected liquid bridges

Fig. 6. Multiple interface system: (a) Two soap bubbles connected by a tube,(b) Two liquid bridges connected by a tube.

\section{Types of Meniscus Boundaries}

I. Soap Film

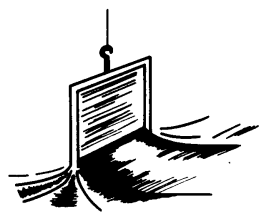

2. Thin Film

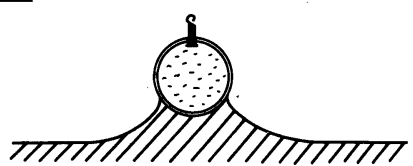

3. Fixed Boundary

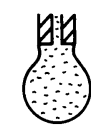

4. Moving Boundary

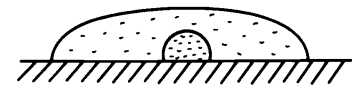

Fig. 7. Types of meniscus boundaries: (a) Soap film, (b) Thin liquid film, (c) Fixed boundary; radius controlled, (d) Moving boundary contact angle controlled.

be supported by a soap film, by a thin liquid film such as with a Wilhelmy plate or directly by a solid such as with a pendant or sessile drop.

The meniscus support is also the theoretical boundary of the meniscus shape derived in the tables. As already noted ${ }^{16}$ the boundary may be controlled by a fixed radius such as obtained with a pendant drop on a circular tip or by a fixed angle of contact such as obtained with a sessile drop. These systems are designated radius and angle controlled respectively. 
INTEGRATION OF THE SHAPE OF A MENISCUS

Integration of the shape of a meniscus is required in order to obtain a set of values of $X$ and $Z$ and other properties such as area and volume at all points on the meniscus. The first method used successfully was the Taylor Series approximation method of Bashforth and Adams ${ }^{4}$ the second method was a geometrical construction method of Kelvin, later developed into a first order method $^{6}$ and the third, and now most usual, is by using the Runge-Kutta method for second order equations. This last method is capable of the greatest accuracy. ${ }^{5}$ The principle of each method is shown in Fig. 8. These integration methods are described fully elsewhere. ${ }^{4-6}$

Integration of the Young-Laplace equation is started at any clearly distinguishable point on the meniscus and for drops this point is at its apex where the two radii of curvature are equal. At each integration step the surface area, the volume and the potential energy increments are also calculated using the equations given in Table 1 . These increments are then summed and recorded.

Rod-in-free-surface menisci present difficulties because they have no natural starting point. This problem was overcome by Huh and Scriven ${ }^{5}$ who showed that as the meniscus shape approaches the free surface of the liquid at very low angles, the value of $Z$ can be obtained from the value of $X$ using the equation

$$
Z=\frac{K_{0}(X) \cdot \tan \phi}{K_{1}(X)}
$$

where $K_{0}$ and $K_{1}$ are Bessel functions of zero and first order respectively and $\phi$ is the meniscus angle. When the value of $X$ is greater than ten, approximate equations for $K_{0}$ and $K_{1}$ are sufficiently accurate to obtain $Z$ as a continuous function of $X$. Huh and Scriven made $\phi=0.5^{\circ}$ but it was found that the greater accuracy required in this study is only achieved when $\phi=<0.02^{\circ}$.

\section{MENISCUS SIZE AND SHAPE FACTORS}

The meniscus possesses a number of properties which are defined in Table 1 . They are $K_{1}$, the meniscus constant which replaces the often used capillary constant; $\beta$ or $\beta^{\prime}$, the shape factor which provides a numerical value to the characteristic shape; and a size parameter which was the radius of curvature at the apex, $b$, of a drop in Bashforth
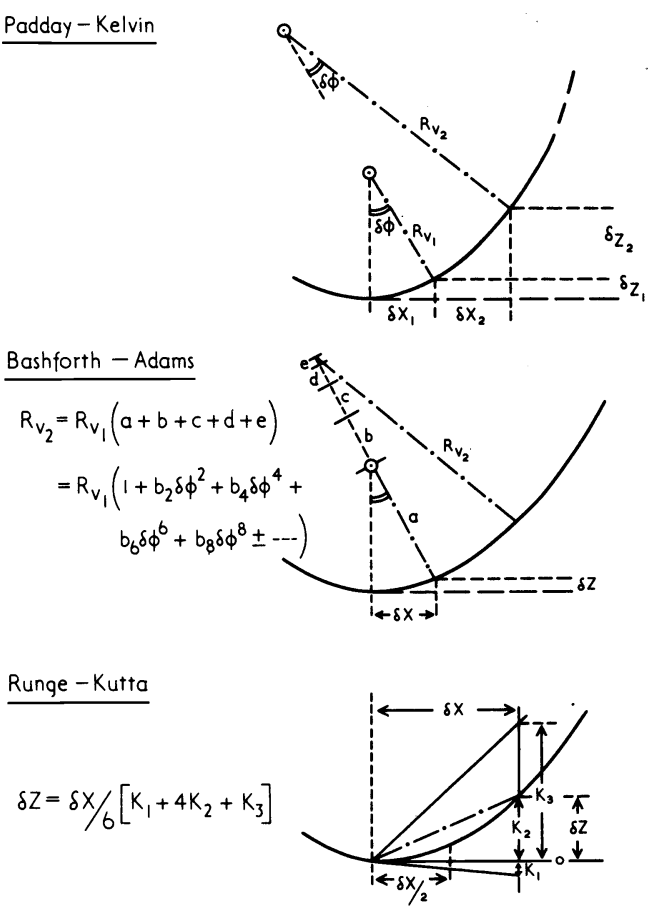

rig. 8. Methods of integrating Young-Laplace equation: (a) Padday-Kelvin method, (b) Bashforth and Adams, (c) RungeKutta.

and Adams' nomenclature and $R_{h}\left(90^{\circ}\right)$ in this study. In bounded meniscus systems $\beta, K$ and $b$ are all explicitly related as shown in Table $1 .\left(\beta^{\prime}, k\right.$ and $R_{h}\left(90^{\circ}\right)$ are also related). Unbounded menisci require a further length parameter, usually $R_{v}\left(90^{\circ}\right)$, to specify the system completely.

Compound menisci such as the pendant lens at a free liquid interface shown in Fig. 5(a) consist of three different meniscus types: namely, a liquid bridge, a rod-in-free surface and a captive bubble all joined together at stable equilibrium. These shapes can in principle be derived theoretically from the tables but great difficulty is found in obtaining the correct conjunction of

Table 1. Shape factors and generating equations for meniscus calculations

\begin{tabular}{lcc}
\hline \multicolumn{2}{c}{ Shape factors } & \\
\hline $\begin{array}{c}\text { Capillary constant } \\
a^{2}=2 \gamma / \rho g \\
K^{2}=\gamma / \rho g\end{array}$ & $\begin{array}{c}\text { Classical } \\
\text { Shape factors }\end{array}$ & 6 \\
Bashforth and Adams & Rayleigh and this study & 7 \\
Padday & $\beta=\frac{\rho g b^{2}}{\gamma}=b^{2} / k^{2}$ & \\
Huh and Scriven & $\beta^{\prime}=\frac{\rho g R_{h^{2}}\left(90^{\circ}\right)}{\gamma}=R_{h^{2}} / k^{2}$ & 9 \\
& $x_{0.5^{\circ}}=X_{0.5} / K$ & 10 \\
B and A & $\operatorname{Equations}^{\circ}$ & \\
Padday & $b / R_{v}+b / R_{h}=\beta Z+2$ & 11 \\
& $k / R_{v}+k / R_{h}=Z / k$ & 12 \\
& $k / R_{v}+k \sin \phi / X=Z^{\prime} / k$ & 13 \\
& $V / K^{3}=\pi X^{2} / k^{2}\left(k / R_{v}-k / R_{h}\right)$ & 14 \\
& $A / k^{2}=2 \pi \int(X / \cos \theta) \mathrm{d} X / k^{2}$ & 15 \\
& $E / k^{4}=\pi \int \mathrm{d}\left[X^{2} / k^{2}\left(k / R_{v}-k / R_{h}\right) Z 0\right]$ & 16 \\
\hline & &
\end{tabular}


equilibrium shapes. Graphical interpolation procedures are often the only method of solving this multiple end point problem to yield the equilibrium and critical properties.

The shape factor, $\boldsymbol{\beta}^{\prime}$, has the additional advantage that it allows the shapes of, and relationships between, the properties of the three types of bounded menisci to be compared. In Fig. 9 a captive bubble, a pendant drop and a rifs meniscus have been plotted with their respective shape factors all equal to unity. The height $Z\left(90^{\circ}\right) / k$ from the free flat surface depends on the meniscus type and it is seen that the neck (the reference point) of the pendant drop is below the free surface (i.e. unduloid) and the other two reference points are above it (i.e. nodoid).

Menisci may be generated at intermediate values of $Z\left(90^{\circ}\right) / k$ and these represent the shapes of unbounded menisci (nodoid or unduloid) of the liquid bridge type that have been discussed previously. ${ }^{6}$

The coordinates and other properties of a meniscus are expressed in dimensionless form by dividing the dimensions of the meniscus by an appropriate power of $K$. Examples of the data obtained by computer integration

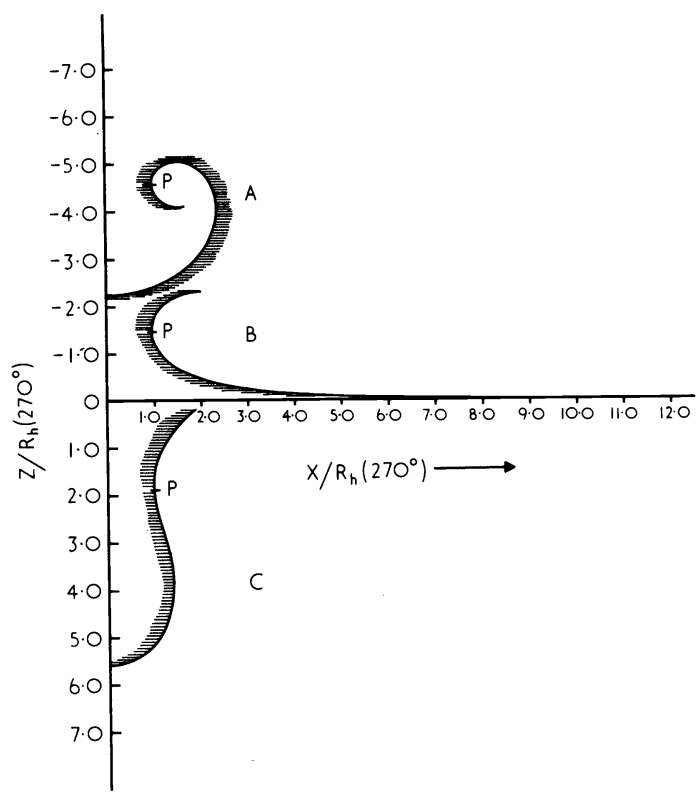

Fig. 9. Relation between bounded menisci $\beta^{\prime}=1.0$. Captive bubble, rod-in-free-surface and pendant drop. are given in Table 2, the upper set representing the properties of a small part of a pendant drop in Bashforth and Adams' base units, $b$, and the lower set the same pendant drop in the base unit, $k$. The full set of data have been published previously as a set of tables. ${ }^{10}$

\section{FREE ENERGY OF A MENISCUS}

The work performed on a system in order to form a meniscus is identified, in general, with two separate and distinctive terms: one is the work performed in changing the surface area of the interfaces present in the system, the other is the work performed by the gravitational force on the system. Providing the meniscus is formed isothermally and reversibly this work may be identified as the Helmholtz free energy, $F$, of formation of the meniscus.

A meniscus is formed by successively adding liquid to it from the bulk phase. At each change in volume the curvature and hence the vapour pressure in equilibrium with the surface, is changed even though by an infinitesimally small amount. For this reason menisci can only bbe formed at constant volume, hence it is necessary to determine the equilibrium using the Helmholtz free energy.

In this study the free energy is derived from the tables on the basis that the work, $W$, done on the system of forming new surface and that of moving liquid from the position of the supporting surface to its equilibrium position within the drop, thus

$$
W=A_{\mathrm{LV}} \gamma_{\mathrm{LV}}+A_{\mathrm{SL}} \gamma_{\mathrm{LV}} \operatorname{Cos} \theta+V \rho g Z_{g}
$$

As the system is always at equilibrium and changes are made infinitely slowly at constant temperature, $W$ equals the Helmholtz free energy.

The free energy is calculated on the assumptions that the liquid in the surface phase has the same density as that in the bulk phase; that the line tension does not contribute to the energy of the system and that the meniscus is sufficiently large for the surface tension to be unperturbed by the size of the system. Though these assumptions are arbitrary, they lead to no significant error in the systems normally under investigation so that the value of $W$ derived from the tables represents the total measured excess free energy of the meniscus.

The condition of stable equilibrium of a meniscus is thus defined as:

$$
\mathrm{d} W / \mathrm{d} \beta^{\prime}=0 ; \quad \mathrm{d}^{2} W / \mathrm{d} \beta^{\prime 2}>0,
$$

\begin{tabular}{|c|c|c|c|c|c|c|c|}
\hline \multirow[b]{2}{*}{ Angle } & \multicolumn{6}{|c|}{$(\beta=-0.500000 ; Z 0 / b=-4.000000)$} & \multirow[b]{2}{*}{$P E / g b^{4}$} \\
\hline & $X / b$ & $Z / b$ & $R_{v} / b$ & $R_{h} / b$ & $V / b^{3}$ & $A / b^{2}$ & \\
\hline 0 & 0 & 0 & 1.0 & 1.0 & 0.0 & 0.0 & 0.0 \\
\hline 5 & 0.087197 & 0.003808 & 1.001432 & 1.000474 & 0.000046 & 0.023932 & 0.000184 \\
\hline 10 & 0.173978 & 0.015236 & 1.005754 & 1.001900 & 0.000726 & 0.095820 & 0.002914 \\
\hline 15 & 0.259926 & 0.034295 & 1.013056 & 1.004278 & 0.003658 & 0.215941 & 0.014742 \\
\hline \multirow[b]{2}{*}{ Angle } & & & $(Z 0 / K=$ & $-2.828 \quad 42$ & & & \\
\hline & $X / k$ & $Z / k$ & $R_{v} / k$ & $R_{h} / k$ & $V / k^{3}$ & $A / k^{2}$ & $E / k^{2}$ \\
\hline 0 & 0.00000 & 0.00000 & $0.707 \quad 11$ & $0.707 \quad 11$ & 0.00000 & 0.00000 & 0.00000 \\
\hline 2 & $0.025 \quad 17$ & 0.00045 & 0.70728 & $0.707 \quad 16$ & 0.00000 & 0.00199 & 0.00002 \\
\hline 4 & 0.05008 & 0.00178 & 0.70777 & 0.70733 & 0.00001 & 0.00789 & 0.00010 \\
\hline 10 & 0.12522 & 0.01116 & 0.71132 & 0.70850 & 0.00028 & 0.04965 & 0.00078 \\
\hline
\end{tabular}

Table 2. Pendant drop profile 
of critical equilibrium as:

$$
\mathrm{d} W / \mathrm{d} \beta^{\prime}=0 ; \quad \mathrm{d}^{2} W / \mathrm{d} \beta^{\prime 2}=0,
$$

of unstable equilibrium as:

$$
\mathrm{d} W / \mathrm{d} \beta^{\prime}=0 ; \quad \mathrm{d}^{2} W / \mathrm{d} \beta^{\prime}<0,
$$

and of non equilibrium as:

$$
\mathrm{d} W / \mathrm{d} \beta^{\prime} \neq 0 .
$$

\section{THE STABILITY OF MENISCI}

In this section the properties of a meniscus corresponding to the criteria for critical equilibrium, just defined, will be sought.

Consider the case of a pendant drop formed at the tip of a circular tube. The growth of such a drop is shown in Fig. 10 , where the volume is plotted as a function of the meniscus angle $\phi$ at the tip. The growth is seen for four different tip radii and all indicate that the drop reaches some maximum volume beyond which further growth within the family of Young-Laplace shapes is no longer possible. The shapes of the menisci for the largest of these tips is shown in Fig. 11.

In a previous publication ${ }^{16}$ it was shown that an energy profile may be constructed which represents the energy the meniscus would possess if it were perturbed at constant tip radius and constant volume, to a different shape that still retained axial symmetry and also a monotonic change in mean curvature with vertical height. Perturbations that are axisymmetric and monotonic with respect to mean curvature may be obtained by manipulat-

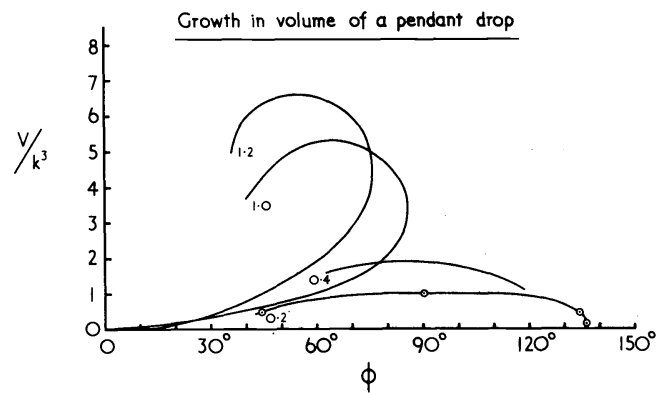

Fig. 10. Volume of a pendant drop shown as a function of its shape during growth. ing the equilibrium tables ${ }^{16}$ and as a result one may describe such a perturbation as in Fig. 12(C) to distinguish them from non-axisymmetric perturbations which are also shown (B).

In this study we made the hypothesis that such axisymmetric perturbations were those of lowest energy and therefore the most damaging. Pitts ${ }^{17}$ has since shown that in the case of pendant drops this was so. The energy profile of a drop is therefore the energy of perturbation plotted as a function of the degree of perturbation.

In Fig. 13, the energy profiles of four pendant drops each of different volume but all with the same tip radius are shown as a function of the degree of perturbation expressed as the height $Z g$. The four separate profiles are of radius-volume controlled pendent drops and it is seen that equilibrium growth of the drop occurs along a line joining the positions at the bottom of each energy trough where $\mathrm{d} W / \mathrm{d} Z g=0$. The drop volume can be successively increased until the critical energy profile is reached and then further increases in volume lead to nonequilibrium

$$
\frac{\text { Work of perturbation }}{\text { Constant } V, \text { Xand } k}
$$

A.

$$
\frac{\text { Unperturbed growth }}{W / \gamma k^{2}=A / k^{2}-V Z g / k^{4}}
$$

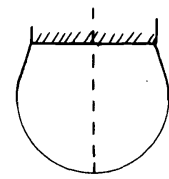

B. Non-axisymmetric perturbed

$$
W^{\prime} / \gamma k^{2}=A^{\prime} / k^{2}-V Z g^{\prime} / k^{4}
$$

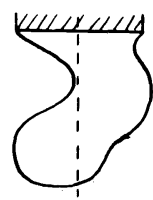

C. Axisymmetric perturbed
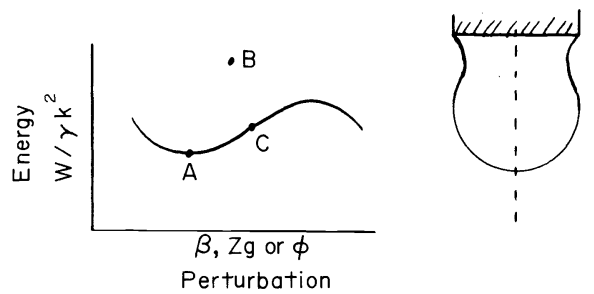

Fig. 12. Types of perturbations of a pendant drop.
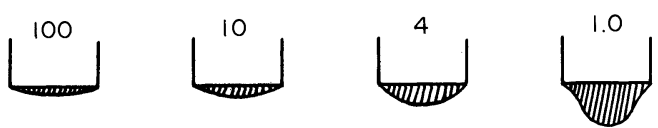

Tip radius

$x / k=1.2$

$\beta$
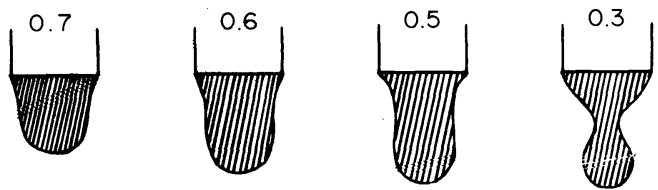

Pendant drop growth

Fig. 11. Shapes of a pendant drop during growth. 


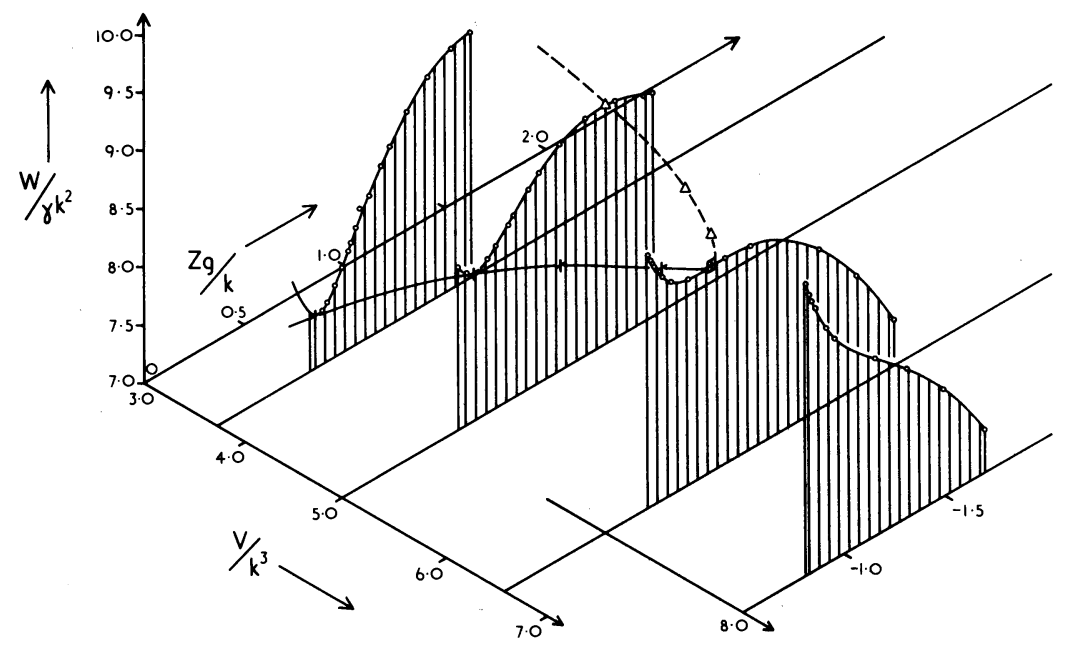

Fig. 13. Multiple energy profiles of a pendant drop as a function of drop volume and degree of perturbation.

conditions. This critical point corresponds to the criteria for critical equilibrium and as pointed out previously corresponds to the maximum volume.

Thus for a given tip radius, $X / k$, and a fixed value of the meniscus constant, $k$, a pendant drop possesses a unique volume at which critical breakaway takes place. It was this critical volume that was sought by earlier investigators who wished to use the drop weight method for surface tension measurement.

The maximum volume condition reached by the pendant drop may be expressed mathematically as

$$
(\mathrm{d} V / \mathrm{d} \beta)_{X, k}=0
$$

and this is the critical condition of a radius-volume controlled pendant drop in its equilibrium state.

The radius-volume controlled pendant drop is only one of four possible methods of growing and perturbing a drop. The other three methods are; angle-volume controlled where the drop is formed at constant contact angle at the under surface of the supporting plate and perturbation is still at constant volume; the radius-pressure controlled pendant drop where the drop is formed at constant tip radius and perturbation is made at constant hydrostatic pressure in the liquid at the tip; and the corresponding angle-pressure controlled drop where the angle of contact is again held constant during growth and perturbation. The critical equilibrium shape of each type of drop is very different.

The critical condition that is reached for each type of pendant drop, sessile drop and rifs meniscus is given in Table 3 and each condition is reached when the appropriate values of both the first and second differentials of the energy, with shape, equal zero.

It now remains to extract critical data from the theoretical tables. Although search and interpolation procedure may be used, by far the simplest with an excellent degree of accuracy is that of the envelope construction technique. This technique consists of plotting a family of curves as in Fig. 14, wherein (in this example) the pendant drop volume is plotted as a function horizontal distance, $X$, of the meniscus from the axis of symmetry at many points on each of several pendant drop curves. An enveloping line is then constructed which contains all the curves as shown in the figure. As all the points plotted are at equilibrium the volumes cannot exceed the critical values hence the enveloping line must represent the required function of the critical conditionin this case the maximum stable volume of a pendant drop.

The theoretical curve derived in Fig. 14 is replotted in Fig. 15 and compared with various equations derived for use with the drop volume method of measuring surface tension. Clearly the theoretical curve cannot agree with measured values of volume because the drop volume derived is that of the whole drop and that measured is only the portion falling away. In order to derive the one from the other further theoretical work is required to describe the process of breaking away.

Even so, the important feature of this study is that unique conditions have been shown to exist in menisci and the numerical values of the properties at these unique conditions have been accurately determined.

Table 3. Conditions for critical equilibrium of menisci

\begin{tabular}{|c|c|c|c|c|}
\hline & $\begin{array}{l}\text { Volume/radius } \\
\text { limited }\end{array}$ & $\begin{array}{l}\text { Pressure + radius } \\
\quad \text { limited }\end{array}$ & $\begin{array}{l}\text { Volume/contact angle } \\
\text { limited }\end{array}$ & $\begin{array}{c}\text { Pressure }+ \text { contact angle } \\
\text { limited }\end{array}$ \\
\hline $\begin{array}{l}\text { Pendant drop } 0= \\
\text { (emergent bubble) }\end{array}$ & $\left(\mathrm{d}\left(V / k^{3}\right) / \mathrm{d} \beta\right)_{T, k, X}$ & $\left(\mathrm{~d}\left(Z_{t} / k\right) / \mathrm{d} \beta\right)_{T, k, X}$ & $\left(\mathrm{~d}\left(V / k^{3}\right) \mathrm{d} \beta\right)_{T, k, \phi}$ & $\left(\mathrm{d}\left(Z_{t} / k\right) / \mathrm{d} \beta\right)_{T, k, \phi}$ \\
\hline $\begin{array}{l}\text { Sessile drop } 0= \\
\text { (captive bubble) }\end{array}$ & $\left(\mathrm{d}\left(V / k^{3}\right) / \mathrm{d} \beta\right)_{T, k, X}$ & $\left(\mathrm{~d}\left(Z_{t} / k\right) / \mathrm{d} \beta\right)_{T, k, X}$ & Always stable & Always unstable \\
\hline Rod-in-free-surface & $\mathrm{d}\left(Z_{t} / k\right) / \mathrm{d} \beta^{\prime}$ & $\mathrm{d}\left(Z_{t} / k\right) / \mathrm{d} \phi$ & $\mathrm{d}\left(Z_{t} / k\right) / \mathrm{d} \beta^{\prime}$ & \\
\hline $\begin{array}{l}\text { (Hole-in-free } \\
\text { surface) }\end{array}$ & $=\frac{1}{\pi X_{d}^{2}}\left(\mathrm{~d} V / \mathrm{d} \beta^{\prime}\right)$ & $=0$ & $\left.=\frac{1}{\pi X_{d}^{2}}\left(\mathrm{~d} V / \mathrm{d} \beta^{\prime}\right)\right\}$ & Always unstable \\
\hline
\end{tabular}




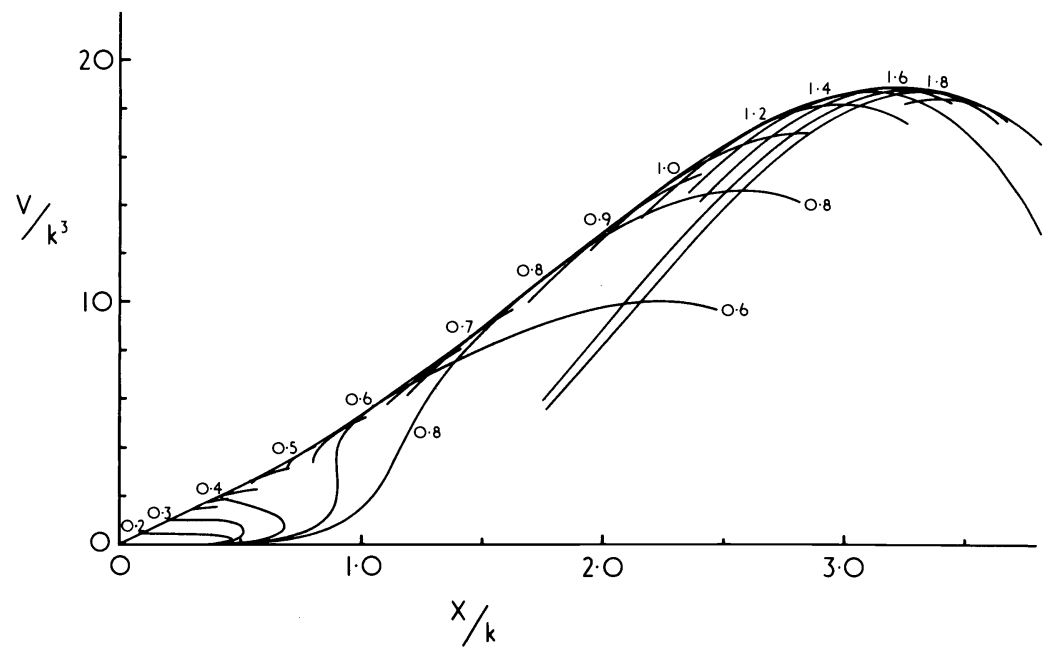

Fig. 14. Maximum volume of a pendant drop as a function of tip radius, obtained by the envelope construction method.

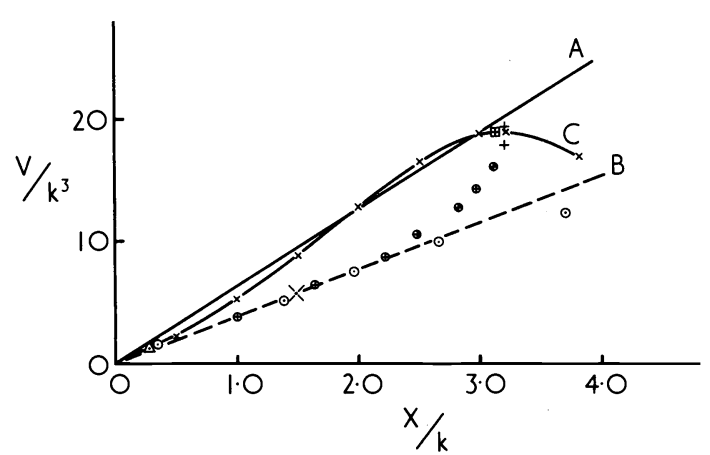

Fig. 15. Pendant drop critical volume as a function of tip radius compared with other studies; A, Tate's equation; ${ }^{1}$ B, Rayleigh's equation; ${ }^{18} \mathrm{C}$, This study; Lohnstein's separating volume; ${ }^{19} ; X$ Lohnsteins critical volume; ${ }^{19}$ Harkins and Brown's drop volumes. ${ }^{20}$

\section{THE MEASUREMENT OF SURFACE TENSION}

Surface tension is invariably measured from the properties of a curved liquid surface, usually a meniscus. Examination of the methods currently available ${ }^{21}$ shows clearly that few of the methods take advantage of determining surface tension at stable equilibrium and at the same time at some unique characteristic point reached during its growth.

Existing methods fall roughly into two groups: those at stable equilibrium such as sessile drop shape, pendant drop shape, capillary rise and Wilhelmy plate methods: and methods involving taking the meniscus past its critical equilibrium such as the drop volume and Du Nöuy ring methods. The supposed advantage of these latter methods is that the drop weight or force reaches a unique critical value. Disadvantages such as premature rupture and the expansion of the surface causing adsorption depletion far outweigh any advantages.

An important feature of most types of menisci is that they possess at least one unique and well-defined characteristic point, during growth, at which a shape or size property reaches a unique maximum or minimum value at stable equilibrium. The properties of the meniscus at the critical point of rupture represent one set of unique conditions but in addition a further set at stable

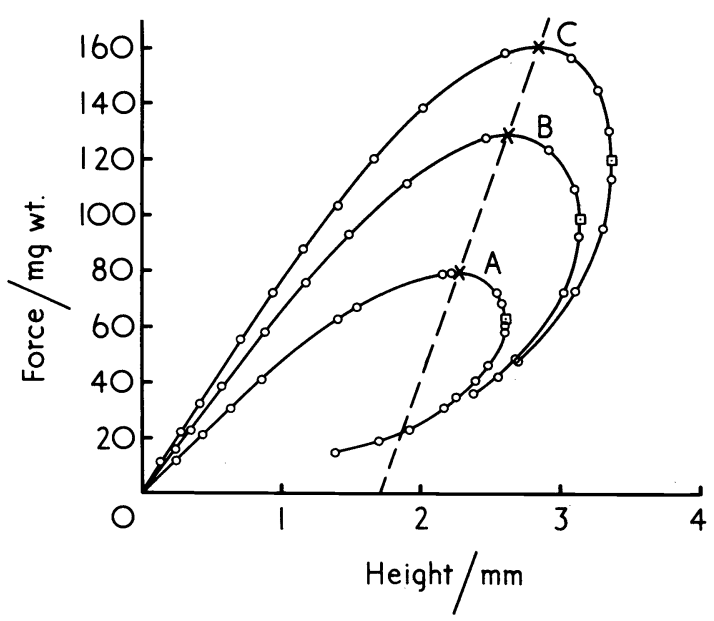

Fig. 16. Force on a rod-in-free-surface meniscus as a function of the growth of the meniscus expressed as its height.

equilibrium often exists which enable the surface tension to be measured with extreme accuracy.

This principle is best explained by studying the example of the properties obtained from the rifs meniscus. In Fig. 16 the force on a rod arising from the volume of liquid supported in a rifs meniscus is plotted as a function of the height of the rod above the free surface. It is well known and proved in a previous study ${ }^{16}$ that critical equilibrium and rupture occurs when the height of the rod reaches a limiting value which is clearly seen in the figure. The curves $\mathrm{A}, \mathrm{B}$ and $\mathrm{C}$ represent here the equilibrium forces on rods of different radii and the points of rupture are indicated by squares $(\square)$; thus parts of each curve stretching back to the origin represent points at stable equilibrium. It is now obvious that the points at which the force on the rod (i.e. maximum volume marked $X$ ) reaches a maximum is at stable equilibrium because they lie on the growth line and are reached well before critical conditions. The stable equilibrium property of this type of meniscus is thus

$$
\left(\mathrm{d}\left(V / k^{3}\right) / \mathrm{d} \beta^{\prime}\right)_{k, X}=0
$$

for a pressure-radius controlled rifs meniscus and is, of 


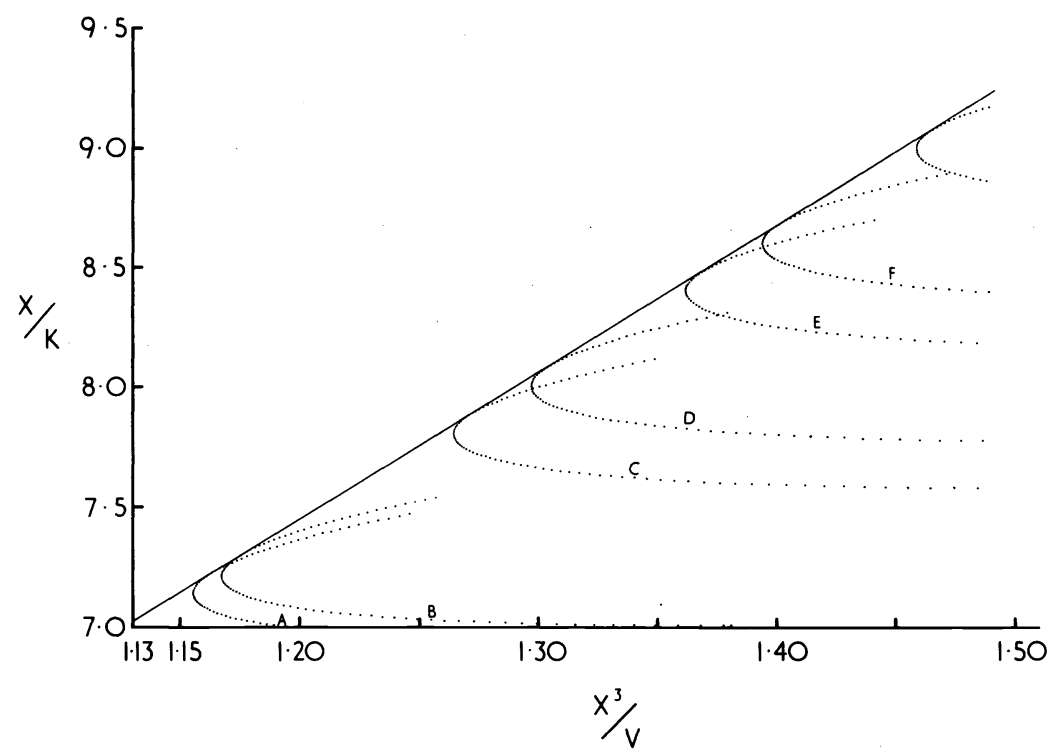

Fig. 17. Envelope construction method to obtain the maximum volume on a rod-in-free-surface meniscus.

course, different from the stability condition given in Table 3 for the same meniscus.

Sessile drops also reach a unique condition, maximum height at a certain stage of their growth, and this condition has been used to measure interfacial tension. ${ }^{22}$ It was shown that a sessile drop could be formed with a contact angle of $180^{\circ}$ (by using a thin film) and that when the maximum drop height is reached, when the drop is only a few centimetres in diameter, the interfacial tension is calculated accurately from the value of the height obtained by theoretical analysis.

The maximum force on the rifs method outlined here and fully described elsewhere ${ }^{23}$ and the maximum force on a sphere-in-a-free surface, also a stable equilibrium method using an unique shape condition, ${ }^{24}$ possess so many advantages that they may well supercede all other methods when they become widely known.

These methods rely on the shape or size property being derived theoretically with sufficient accuracy. Although computer interpolation procedures may be used to obtain accurate theoretical values for the maximum volume of a rifs meniscus, the envelope construction method is both accurate and easy to operate and gives the required unique maximum volumes (in the case of the rifs meniscus) as a function of rod radius as shown in Fig. 17. In this figure greater accuracy and near linearity is obtained by plotting the volume as the reciprocal function $X^{3} / V$.

Attempts have been made to calculate the relationship between the maximum volume of a rifs meniscus and the rod radius but in general such calculations either are not sufficiently accurate or only apply under a very limited set of conditions such as with very small or very large rods. The maximum force, expressed as a volume, is expressed by the equation

$$
V / k^{3}=\pi X^{2} Z / k^{3}+2 \pi X(\sin \phi) / k .
$$

With large rods, $\phi$ tends to zero and the first term predominates so that

$$
V \mid k^{3} \bumpeq \pi X^{2} Z / k^{3} \bumpeq 2 \pi X^{2} / k^{2}
$$

and with small rods, $\phi$ tends to $90^{\circ}$ so that the second term predominates and

$$
V / k^{3} \bumpeq 2 \pi X / k
$$

Surface tension methods can be classified in a way similar to that used to classify stability criteria. Surface tension may be measured with a cylindrical meniscus (Wilhelmy plate) or with a axisymmetric meniscus (capillary rise): also it can be measured by a radius controlled equilibrium (rifs and pendant drop methods) and by a radius-pressure controlled equilibrium (maximum bubble pressure method), among many others. The classification of these methods is important because it enables the correct data to be extracted from the equilibrium tables to give a theoretical value to the constant relating surface tension to the measured property. By doing this one is able to arrive at absolute values of surface tension that are free of arbitrary constants and corrections.

However even when such constants are determined theoretically experimental error arises from the weight of a wetting film and the solid may not be exactly zero because the wetting film may possess effective surface tension value different from the bulk. These difficulties however are outweighed by the advantages of using unique shape conditions to measure surface tension. The advantages include the ability to measure and follow dynamic and ageing effects and the ability to choose from among several methods according to the nature of the system.

\section{REFERENCES}

${ }^{1}$ T. Tate, Phil. Mag. 27, 176 (1864).

${ }^{2}$ T. Young, Miscellaneous Works (edited by G. Peacock), Vol. 1, p. 149. Murray, London (1804).

${ }^{3}$ P. S. de Laplace, Méchanique Celèste. Suppl au Xe livre. Paris, Gauthier (1805).

${ }^{4} \mathrm{~F}$. Bashforth and J. C. Adams, An Attempt to Test the Theory of Capillary Action. Cambridge University Press (1883).

${ }^{5}$ C. Huh and L. E. Scriven, J. Colloid Interface Sci. 30, 323 (1969).

${ }^{6} \mathrm{~J}$. F. Padday, Phil. Trans. Roy. Soc. Lond., A269, 265 (1971).

${ }^{7}$ D. W. G. White, A suppl. to the tables of Bashforth and Adams. Internal report P.M. 1-67-4. Dept. of Energy, Mines and Resources, Ottawa, Canada (1967). 
${ }^{8}$ H. M. Princen, Surface and Colloid Science. (edited by E. Matejevic) Vol. 2, p. 11. Wiley, London (1969).

'J. F. Padday, J. Electroanal. Chem. 37, 313 (1972).

${ }^{10} \mathrm{~J}$. F. Padday, Tables of meniscus shape published as a set of microfisches obtainable from Kodak Limited, Research Division, Wealdstone, Harrow.

${ }^{11} \mathrm{P}$. Concus and R. Finn, Proc. Nat. Acad. Sci. (Washington), 63, 292 (1969).

${ }^{12}$ H. M. Princen, J. Colloid Interface Sci. 30, 359 (1969).

${ }^{13}$ J. F. Padday and A. Pitt, J. Colloid Interface Sci., 38, 323 (1972).

${ }^{14}$ A. C. Zettlemoyer, M. P. Aronson and M. C. Wilkinson, J. Colloid Interface Sci. 37, 498 (1971). Ibid. 52, 1 (1975).

${ }^{15}$ J. Plateau, Statique Experimentale et Theorique des Liquides. Gauthier, Paris (1873).
${ }^{16}$ J. F. Padday and A. Pitt, Phil. Trans. Roy. Soc. Lond. 275A, 489 (1973).

${ }^{17}$ E. Pitts, J. Inst. Maths. Applic. 17, 387-397 (1976).

${ }^{18}$ Lord Rayleigh, Phil. Mag. 48, 321 (1899).

${ }^{19}$ T. Lohnstein, Annln. Phys. 21, 1030 (1907); ibid. 22, 767 (1907); ibid. 84, 410 (1913).

${ }^{20}$ W. D. Harkins and F. E. Brown, J. Am. Chem. Soc. 41, 503 (1919).

${ }^{21}$ J. F. Padday, Surface and Colloid Science (edited by Matejevic), Vol. 1, p. 101. Wiley, London (1967).

${ }^{22}$ J. F. Padday and A. Pitt, Proc. Roy. Soc. A329, 421 (1972).

${ }^{23}$ J. F. Padday, R. Pashley and A. Pitt, J. Chem. Soc., Farad. Trans. I, 75, 1919 (1975).

${ }^{24}$ C. Huh and S. G. Mason, J. Colloid Interface Sci. 47, 271 (1974). 